\title{
Music Recommendation System through Internet for Improving Recognition Ability Using Collaborative Filtering and Impression Words
}

\author{
Yasunari Yoshitomi, Taro Asada, Ryota Kato, Yuuki Yoshimitsu, Masayoshi Tabuse \\ Graduate School of Life and Environmental Sciences, Kyoto Prefectural University, \\ 1-5 Nakaragi-cho, Shimogamo, Sakyo-ku, Kyoto 606-8522, Japan \\ E-mail: \{yoshitomi, tabuse\}@kpu.ac.jp, \{t_asada,r_kato\}@mei.kpu.ac.jp \\ Noriaki Kuwahara \\ Graduate School of Science and Technology, Kyoto Institute of Technology, \\ Matsugasaki, Sakyo-ku, Kyoto 606-8585, Japan, \\ E-mail: nkuwahar@kit.ac.jp \\ Jin Narumoto \\ Graduate School of Medical Science, Kyoto Prefectural University of Medicine, \\ Kajii-cho, Kawaramachi-Hirokoji, Kamigyo-ku, Kyoto 602-8566, Japan \\ E-mail: jnaru@koto.kpu-m.ac.jp
}

\begin{abstract}
In our previously reported system, after the first music recommendation process using collaborative filtering was terminated, the second recommendation process based on the scores for impression words finds the most similar music to the successfully recommended music among music that has not yet been recommended. In the present study, based on our previously reported system, we propose a system for music recommendation through the Internet using a videophone system (Skype). The effectiveness of the proposed system is verified.
\end{abstract}

Keywords: Collaborative filtering, Music recommendation, Music therapy, Impression word, Internet, and Skype.

\section{Introduction}

In Japan, the average age of the population has been increasing, and this trend is expected to continue. This trend is more remarkable in rural areas. Recently, music therapy has been used to improve the recognition ability of people, particularly older people. Music therapy may be more effective if music that is liked by an individual is adopted. We have been developing a music recommendation system aimed at improving recognition ability. $^{1,2}$
In the present study, based on our previously reported system ${ }^{2}$, we propose a system for music recommendation through the Internet using a videophone system $\left(\right.$ Skype $\left.^{3}\right)$, and verify the effectiveness of the proposed system.

\section{Music Recommendation Method Using Impression Words}

We use ten pairs of impression words (Table $1^{4}$ ). As an example, we show a user scores for one pair of impression words (quiet - busy), and the user scores the word pairs according to seven levels, which are then 
transformed to three levels, as shown in Table $2^{1}$. A music database was created in which all songs in the database were assigned scores, $i$ ( $-3 \leq i \leq 3)$, for each pair of impression words evaluated by the participants. Fig. 1 shows a flowchart of the music recommendation based on impression words. When music that has not been recommended to a user has the same scores, with the exception of " 0 ", as that of at least one recommended piece of music that has been evaluated highly by the user on the three-level score for at least five impression words, the music is treated as having been positively evaluated by the user. In contrast, when music that has not been recommended to the user has the same scores, with the exception of " 0 ", as that of another piece of music that has been recommended to the user and has been negatively evaluated by the user on the three-level score for at least seven impression words, the music is treated as having been negatively evaluated by the user. In Fig. 1, when none of the not recommended music receives a positive evaluation by the user, another recommendation is performed by using the subjective estimations of all users whose subjective estimations are stored in the database.

Table 1. Pairs of impression words. ${ }^{4}$

\begin{tabular}{c}
\hline quiet - busy \\
\hline bracing - heavy \\
\hline easy - uneasy \\
\hline cheerful - gloomy \\
\hline refreshing - depressing \\
\hline happy - sad \\
\hline comforting - harmful \\
calm - elevating \\
clean - dirty \\
\hline magnificent - superficial
\end{tabular}

Table 2. Scores for pairs of impression words, quiet - busy. ${ }^{1}$

\begin{tabular}{ccc}
\hline Score & Three-level score & impression \\
\hline 3 & -1 & very busy \\
\hline 2 & -1 & busy \\
\hline 1 & 0 & slightly busy \\
\hline 0 & 0 & neutral \\
\hline-1 & 0 & slightly quiet \\
\hline-2 & 1 & quiet \\
\hline-3 & 1 & very quiet \\
\hline
\end{tabular}

It is expressed by "with highest similarity" in Fig. 1 that the music has the highest proportion of the same three-level scores except " 0 " as that of other music recommended to the user and given a positive evaluation by the user among the music not yet recommended to the user. In Fig. 1, the "set of music with a similarity to the recommended music based on impression words" (MSRIW) is decided by using at least seven pairs of impression words in the case of a negative evaluation.

\section{Proposed System}

Fig. 2 shows an overview of the proposed system. The function of Skype ${ }^{3}$, which is described in Fig. 2, makes it possible to simultaneously send music, voice, and image through The Internet to the user of our music recommendation system. At the same time, using Skype $^{3}$, we can communicate through The Internet with the user during music recommendation by our system. After the user listens to each music recommended by our system, we ask the user whether he or she likes the recommended music or not. The answer of the user is input to our music recommendation system, and then will be used for selecting music for the future users by our recommendation system. When we constructed the system shown in Fig.2, we referred the Web site ${ }^{5}$ for setting PC, devises, and Skype ${ }^{3}$.

Fig. 3 shows a flowchart of our previously reported system, which combines collaborative filtering and our music recommendation process based on impression words.

In the proposed system, the recommendation process using collaborative filtering is terminated when the number of users is zero in the reference list of users showing exactly the same evaluation for the recommended music as that of the user up to that moment. Then, the recommendation process performs by finding out the most similar music, from the viewpoints of three-level scores except "0" on impression words, to that successfully recommended among music not yet recommended. The proposed system recommends music stored in the database to user $u$, as shown in Fig. 3. The recommendation process using the proposed system is terminated when the number of recommended songs reaches the upper limit $K$, which has been decided previously. Just before finishing the recommendation process, the database of users with subjective estimations of the music is 


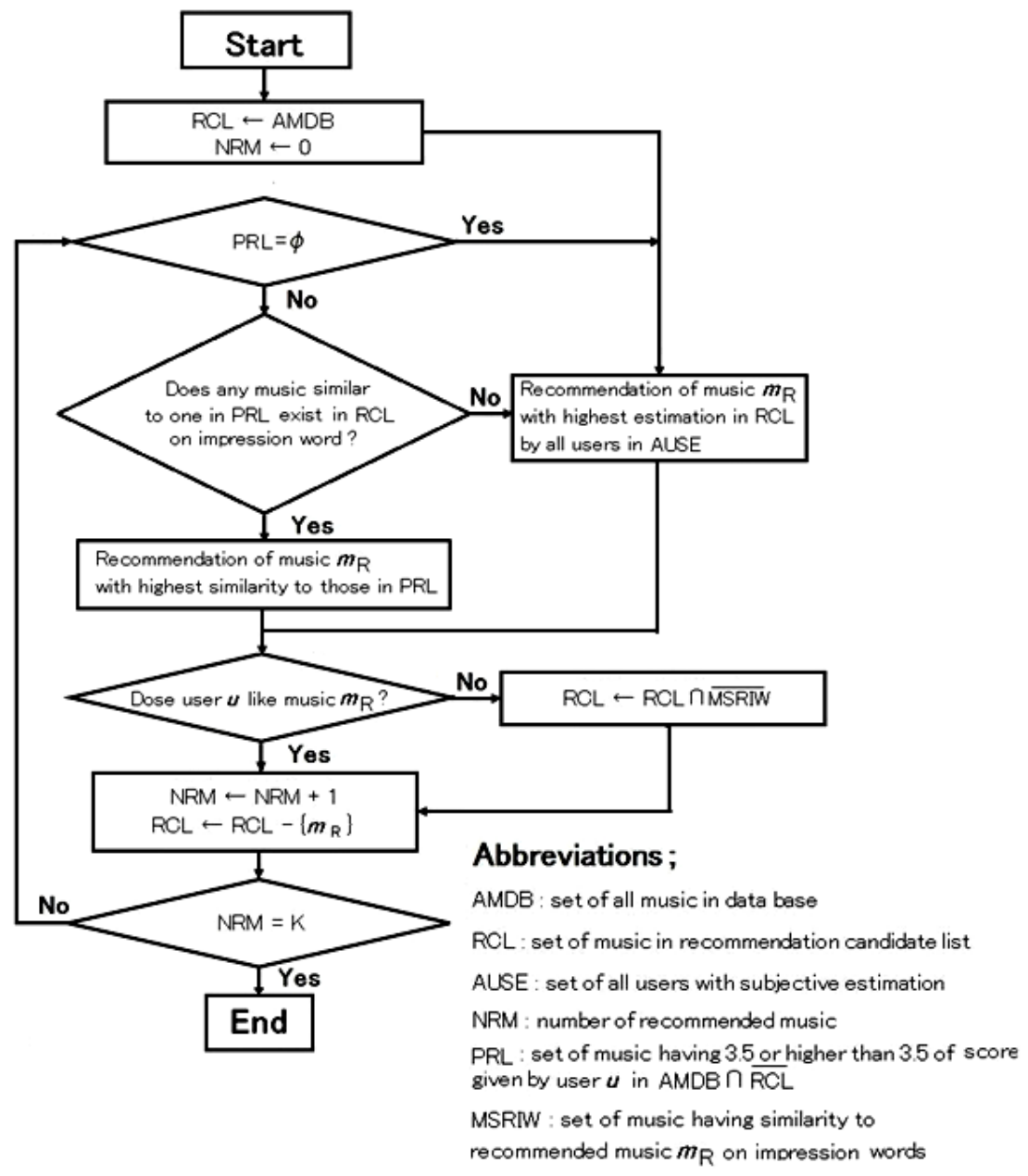

Fig. 1. Flowchart of music recommendation by using impression words. ${ }^{1}$

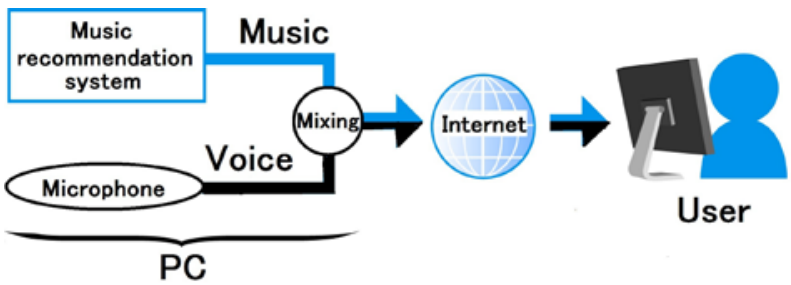

Fig.2. Overview of the proposed system.

updated by adding the subjective estimations of the user

for whom the proposed system recommends music. ${ }^{2}$ 


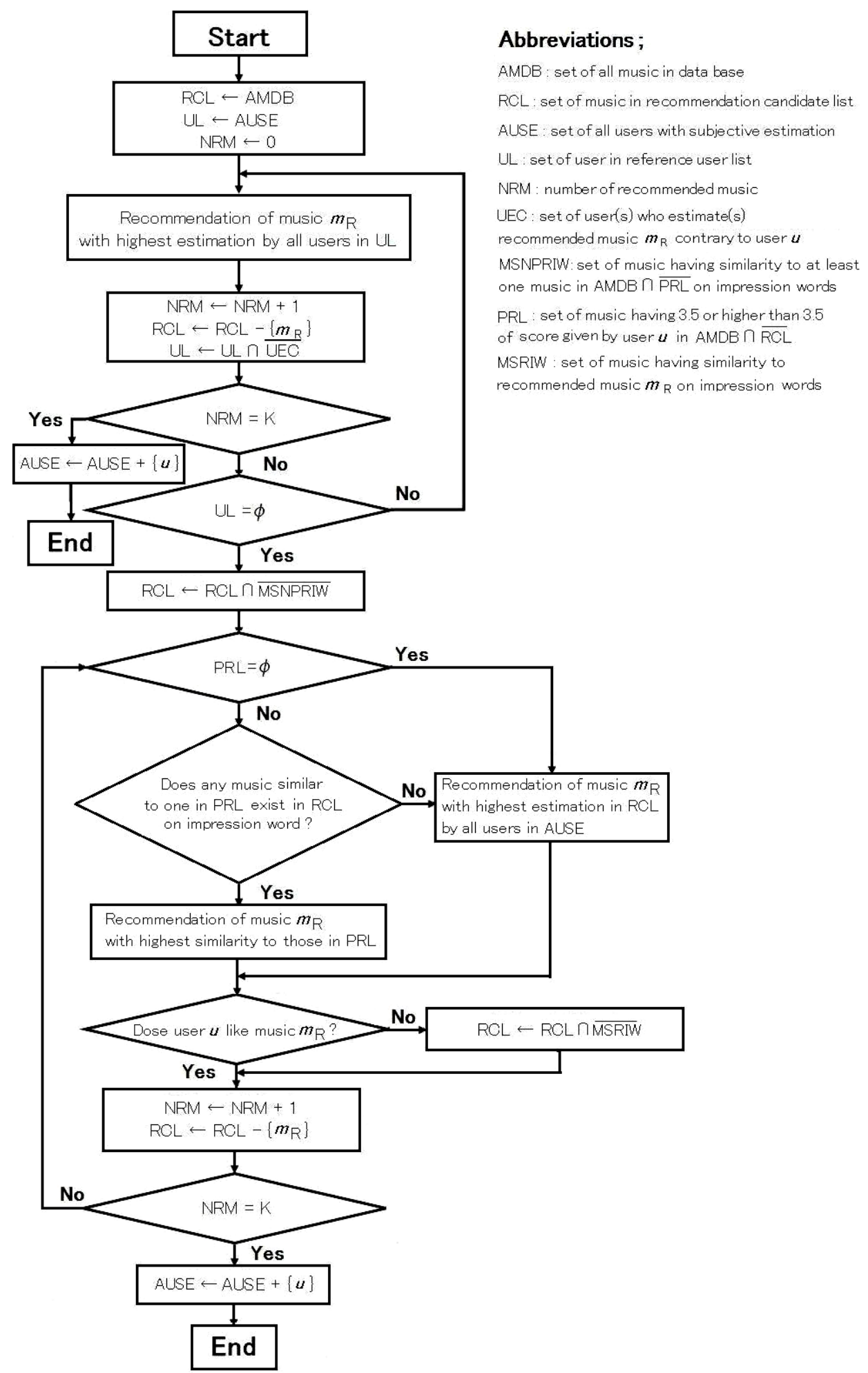

Fig. 3. Flowchart of music recommendation used in the proposed system. ${ }^{2}$ 
In the flowchart shown in Fig. 3, the estimation of user $u$ for song $m_{R}$ is set to 1 when the score of user $u$ for song $m_{R}$ is 4 or 5 ("slightly like" or "favorite"), and is set as 0 when the score is 1 to 3 ("dislike," "slightly dislike," or "neutral”). We used ten pairs of impression words (Table $1^{4}$ ). As an example, we showed the user scores for one pair of impression words (quiet - busy). Users scored the word pairs according to seven levels, which are then transformed to three levels, as shown in Table $2^{1}$.

\section{Performance Evaluation}

\subsection{Conditions}

Since older people tend to prefer children's songs ${ }^{6}$, we selected a $\mathrm{CD}^{7}$ described as an anthology of older songs enjoyed by older people with dementia and selected 52 songs on the $\mathrm{CD}$ that were also included in a music textbook database ${ }^{8}$ for elementary schools. The Japanese titles of the 52 songs are listed in Table 3.

Table 3. List of songs used in the present study.

\begin{tabular}{|l|l|l|l|}
\hline Harugakita & Teruterubozu & Mushinokoe & Hiraitahiraita \\
\hline Takibi & Natsuwakinu & Soranbushi & Zuizuizukkorobashi \\
\hline Akaikutsu & Tanabata & Harunoogawa & Antagatadokosa \\
\hline Usagi & Natsunoomoide & Muramatsuri & Usagitokame \\
\hline Sakura & Warewauminoko & Kutsuganaru & Umi (Chinese character) \\
\hline Hana & Umi (Hiragana) & Yuuhi & Urashimatarou \\
\hline Koinobori & Kintarou & Kakashi & Momotarou \\
\hline Seikurabe & Akatonbo & Furusato & Hanasakajiji \\
\hline Fujinoyama & Yuuyakekoyake & Yuki & Ushiwakamaru \\
\hline Chatsumi & Koujyounotsuki & Oshougatsu & Ureshiihinamatsuri \\
\hline Kisha & Oborozukiyo & Katatsumuri & Nanatsunoko \\
\hline Amefuri & Tsuki & Kagome & Ichigatsutsuitachi \\
\hline Ame & Momiji & Touryanse & Dongurikorokoro \\
\hline
\end{tabular}

In order to evaluate the music recommendation method, all 52 of the selected songs in the database were assigned scores, $s(1 \leq s \leq 5)$, by 12 subjects of different ages (13-19 years: one subject, 20-29 years: six subjects, 50-59 years: five subjects). We used 15 as the value for $K$ in the evaluations of the proposed method. In addition, all 52 of the selected songs in the database were assigned scores, $i(-3 \leq i \leq 3)$, for each pair of impression words by five subjects of different ages (20-29 years: three subjects, 40-49 years: one subject, 50-59 years: one subject). The subject in his fifties also assigned the scores, s. The average score $i$ obtained from the five subjects for each pair of impression words was used as the score $i$ for the performance evaluation. The 15 songs having threelevel scores other than " 0 " for one impression word at most were not recommended in the recommendation process based on impression words because these songs did not have distinct characteristics from the viewpoint of impression words.

Updating of the AUSE, which is the set of all users with subjective estimations in Fig. 3, was effective in improving the accuracy of the recommendation and increasing the number of recommended songs. ${ }^{2}$ Therefore, in order to improve the performance of the proposed system, we used a face-to-face system that updated the AUSE for 44 users before this experiment was performed.

For programming, we used Visual C++ 6.0 (Microsoft) on a PC (Dell Latitude E6599, CPU: Intel Core 2 Duo P8700 2.54 GHz, main memory: 4.00 GB, and OS: Windows 7, Microsoft) for the experiment. We used a videophone system (Skype) to send music to users over the Internet. The proposed system was set up at Kyoto Prefectural University in Kyoto City located in the southern part of Kyoto Prefecture in Japan. The participants for this experiment resided in Kyotango City in the northern part of Kyoto Prefecture, Japan. Using Skype over the Internet, 10 older users, referred to as users 1 through 10, of different ages (70-79 years: three subjects, 80-89 years: six subjects, 90-99 years: one subject) participated in the experiment on user-byuser updating for the AUSE. All of the users were female. Users 3, 4, and 8 were the same person and users $5,6,7$, and 10 were also the same person.

\subsection{Results and discussion}

Fig. 4 shows the performance of the proposed system, with the updating of the AUSE for users 1 through 10 .

The mean value of the number of recommended songs for users 1 through 10 was 14.9 per trial. The mean value of the recommendation accuracy for 10 trials by the users 1 through 10 was $90.6 \%$.

The impression words used in the present study were selected based on the linguistic method ${ }^{4}$. The questionnaire was completed by 100 subjects for 80 pieces of classical music and the impression words used in the present study were demonstrated to have very low correlation with each other. ${ }^{4}$ For details on the selection of impression words, see Reference 4 . The impression words used in the present study are not related to the 

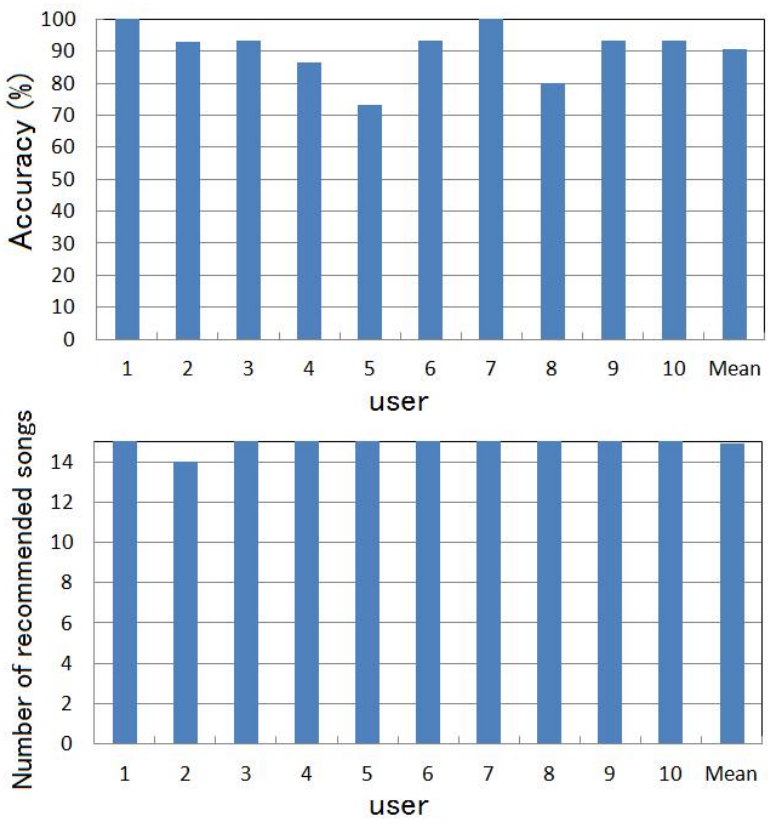

Fig. 4. Performance of the proposed system. Upper: recommendation accuracy, Lower: number of recommended songs.

acoustic features of music, such as tempo, tonality, rhythm, and harmony.

\section{Conclusion}

We have proposed a system for music recommendation over the Internet using a videophone system (Skype). The effect of this system was verified in the present study. The recommendation accuracy of the proposed system was $90.6 \%$ for 10 trials by five elderly subjects. The average number of recommended pieces of music for subjects by the proposed system was 14.9 per trial. In the future, we intend to increase the number of user evaluation scores in the database and apply the proposed system to older individuals and/or to individuals with cognitive impairment.

\section{Acknowledgment}

We would like to thank all of the subjects who participated in the experiments. The present study was supported in part by SCOPE (122307003) of the Ministry of Internal Affairs of Communications of Japan.

\section{References}

1. C. Koro, Y. Yoshitomi, T. Asada, and S. Yoshizaki, Music recommendation aimed at improving recognition ability using collaborative filtering and impression words, in Proc. 17th Int. Symp. on Artificial Life and Robotics, ed. M. Sugisaka (Japan, Beppu, 2012), pp. 222-225.

2. S. Yoshizaki, Y. Yoshitomi, C. Koro, and T. Asada, Music recommendation hybrid system for improving recognition ability using collaborative filtering and impression words, J. Artif. Life and Robotics 18(1-2) (2013) 109-116.

3. Skype, http://www.skype.com/ Accessed 5 November 2013.

4. T. Kumamoto and K. Ohta, Design of scales to represent user's impressions of a music piece for use in a musicretrieval system (in Japanese), IPSJ SIG Notes, 2001-NL147(6), (2002), 35-40.

5. http://www18.atwiki.jp/live2ch/ Accessed 9 January 2015.

6. T. Takahashi, Research report on songs familiar to people advanced in years (in Japanese), J. Japanese music therapy associate 15(1) (1997) 68-75.

7. T. Akahoshi, Good old anthology enjoyable for people advanced in years and troubled with dementia (in Japanese) ( Kirara shobo, Tokyo, 2009).

8. Music textbook database for elementary school by Kanagawa prefectural education center (in Japanese), http://kjd.edu-ctr.pref.kanagawa.jp/daizai_music/ Accessed 11 March 2013. 